\title{
Corticostriatal dynamics underlying components of binge-like eating in mice
}

Britny A. Hildebrandt, Ph.D. ${ }^{1}$, Hayley Fisher, Ph.D. ${ }^{1}$, Zoe LaPalombara, Ph.D. ${ }^{1}$, Michael E. Young, Ph.D. ${ }^{2}$, Susanne E. Ahmari, M.D., Ph.D. ${ }^{1,3,4}$

${ }^{1}$ Department of Psychiatry, University of Pittsburgh School of Medicine, Pittsburgh, PA, USA

${ }^{2}$ Department of Psychological Sciences, Kansas State University, Manhattan, KS, USA

${ }^{3}$ Center for Neuroscience, University of Pittsburgh, Pittsburgh, PA, USA

${ }^{4}$ Translational Neuroscience Program, Department of Psychiatry, University of Pittsburgh, PA, USA

Corresponding Author:

Britny Hildebrandt, Ph.D.

University of Pittsburgh School of Medicine

Department of Psychiatry

Bridgeside Point II, Suite 223

450 Technology Drive

Pittsburgh, PA 15219

E-mail: britny.hildebrandt@gmail.com

Running Head: $\quad$ In vivo dynamics underlying BE

\section{Submitted: $\quad$ Biorxiv}

Word Count: $\quad 3,884$ 


\begin{abstract}
Binge eating $(\mathrm{BE})$ is a maladaptive repetitive feeding behavior present across nearly all eating disorder diagnoses. BE is associated with poor psychosocial outcomes (e.g., suicidal ideation) and increased risk for obesity. Despite the substantial negative impact of $B E$, its underlying neural mechanisms are largely unknown. Many other repetitive behavior disorders (e.g., obsessive compulsive disorder) show dysfunction within corticostriatal circuitry. Additionally, previous pre-clinical and clinical work has highlighted an imbalance between goal-directed and habitual responding in BE. The aim of the current study was to longitudinally examine in vivo neural activity within corticostriatal regions associated with habitual behavior- the infralimbic cortex (IL) and dorsolateral striatum (DLS)- in a robust pre-clinical model for BE. Female C57BL/6 mice ( $N=32$ ) were randomized to receive: 1 ) intermittent (daily, 2-hour) binge-like access to palatable food (BE mice), or 2) continuous, non-intermittent (24-hour) access to palatable food (non-BE mice). In vivo calcium imaging was performed via fiber photometry at the baseline timepoint and after 4 weeks (chronic timepoint) of engagement in the model for BE. Feeding behaviors (feeding bout onset, offset) during the recordings were captured using contact lickometers which generated TTL outputs for precise alignment of BE behavior to neural data. Results in the IL showed no specific changes in neural activity related to BE. However, BE animals showed decreased DLS activity from the baseline to chronic timepoint at feeding onset and offset. Additionally, BE mice had significantly lower DLS activity at feeding onset and offset during the chronic time point compared to non-BE mice. These results point to a role for DLS hypofunction in chronic $\mathrm{BE}$, highlighting a potential target for future treatment intervention.
\end{abstract}




\section{Corticostriatal dynamics underlying BE 3}

\section{Introduction}

Binge eating $(\mathrm{BE})$ is a chronic and repetitive eating disorder behavior that is present across nearly all eating disorder diagnoses (i.e., anorexia nervosa binge-purge subtype, bulimia nervosa, binge eating disorder, American Psychiatric Association, 2013) and is also widely prevalent in the general population. BE is strongly associated with elevated rates of obesity (Spitzer et al., 1993; Stice, Cameron, Killen, Hayward, \& Taylor, 1999; Stice, Presnell, \& Spangler, 2002), poor psychosocial outcomes (e.g., suicidal ideation, Conti et al., 2017; Telch \& Stice, 1998), and significant medical consequences (e.g., type II diabetes, Herpertz et al., 1998). Despite the substantial negative impact of BE, current treatments for BE and bingerelated eating disorders are often inadequate, rates of relapse and persistence of eating disorder symptoms are high (Keel, Dorer, Franko, Jackson, \& Herzog, 2005; Olmsted, MacDonald, McFarlane, Trottier, \& Colton, 2015), and there has been little success with pharmacological treatments developed specifically for BE (Goracci et al., 2015; Vocks et al., 2010). Together, this underscores a critical need for research aimed at identifying the neural mechanisms contributing to $B E$, which could lead to the development of more effective biologically informed treatments.

Aberrant activity within corticostriatal circuitry has been found in psychiatric conditions associated with maladaptive repetitive behaviors such as obsessive compulsive disorder and substance use disorders (Burguiere, Monteiro, Mallet, Feng, \& Graybiel, 2015; George \& Koob, 2010; Graybiel \& Rauch, 2000; Harrison et al., 2009; Kalivas, 2008). Models for these disorders have highlighted dysfunction in the balance between goal-directed and habitual behavior, suggesting increased reliance on circuitry associated with habit after more chronic durations of illness (Gillan et al., 2011; Hogarth, Balleine, Corbit, \& Killcross, 2013). However, despite evidence for corticostriatal dysfunction underlying other psychiatric conditions with prominent repetitive behaviors, there is little work directly investigating corticostriatal circuit activity in BE.

Clinical BE research has suggested that a transition to compulsive/habitual action may be present in binge related disorders (Moore, Sabino, Koob, \& Cottone, 2016; Pearson, Wonderlich, \& Smith, 2015), and that aberrant function within corticostriatal circuitry may underlie BE behavior (Kessler, Hutson, Herman, \& Potenza, 2016). However, to date, most clinical studies attempting to investigate habit/goal-directed action and the associated neural underpinnings of BE have mostly targeted obese, non-BE populations (Horstmann et al., 2015; Janssen et al., 2017). Pre-clinical work has found that male rats with a history of chronic BE show increased habitual responding during devaluation and increased activity (measured using c-Fos) in corticostriatal regions associated with habit - the infralimbic cortex (IL) and 
dorsolateral striatum (DLS) (Furlong, Jayaweera, Balleine, \& Corbit, 2014). Together, these findings suggest that chronic binge-like eating may lead to habitual behavior and corresponding aberrant activity within corticostriatal circuitry.

While findings from previous work provide evidence that a history of BE leads to dysregulation of corticostriatal circuits related to habitual performance, to date, there have been no in vivo examinations of activity within brain regions associated with habitual behavior during binge-like eating episodes. The aim of this study was to fill this gap by identifying corticostriatal dynamics associated with food intake during BE using in vivo fiber photometry in the IL and DLS in BE and non-BE mice. We hypothesized that after a chronic duration of binge-like eating, BE mice would show changes in neural activity within regions associated with habitual behavior (IL, DLS) during specific feeding behaviors (feeding onset, feeding offset) compared to non-BE mice, suggesting aberrant corticostriatal function after chronic BE.

\section{Methods and Materials}

$\underline{\text { Animals }}$

Thirty-two adult female C57BL/6 mice (from Jackson Laboratories or in house breeding) were used for all experiments. Mice were group housed with 3-5 mice per cage and had ad libitum access to food and water for the entirety of the study. Animals were maintained on a 12/12-hour light-dark cycle (lights off at 7:00 AM; on at 7:00PM). All experiments were approved by the Institutional Animal Use and Care Committee at the University of Pittsburgh in compliance with National Institutes of Health guidelines for the care and use of laboratory animals.

\section{Stereotaxic Surgeries}

Animals were allowed to acclimate to the light-dark cycle for approximately 10 days prior to surgery. On the day of surgery, mice were anesthetized using $5 \%$ isoflurane combined with oxygen for the duration of surgery. Burr holes were drilled unilaterally in the left hemisphere over the target regions for virus injection and fiberoptic implant. A unilateral injection of $350 \mathrm{~nL}$ AAV1-Synapsin.NES-jRCaMP1b.WPRE.SV40 (titer 2.0×1012; from Addgene) and 500 nL AAV9Synapsin-GCaMP6m-WPRE-SV40 (titer 2.7×1012; from Addgene) were injected into the IL (AP: 2.28, ML: 0.3, DV: -2.15) and DLS (AP: 0.9, ML: 2.6, DV: -2.3) respectively. Following viral injection, optical fibers (NA $=.37$ ) were implanted into the IL and DLS using the same coordinates noted above. After completion of the surgery, mice were monitored until fully 


\section{Corticostriatal dynamics underlying BE 5}

recovered from anesthesia, and then remained in their home cage for 3 weeks to allow for recovery and viral incubation.

\section{Binge Eating Paradigm}

The BE paradigm is based on previous work in male rats (Furlong et al., 2014), and was adapted and tested in female mice in our laboratory. We chose to focus our work on female mice given the well-established sex difference in BE behavior in both humans (Klump, Culbert, \& Sisk, 2017) and rodents (Culbert, Sinclair, Hildebrandt, Sisk, \& Klump, 2018; Klump, Racine, Hildebrandt, \& Sisk, 2013). Additionally, pilot work in our laboratory using this paradigm showed significantly higher PF intake in female compared to male mice, providing additional evidence for sex differences in BE in this model.

For the current study, mice were randomized to one of two feeding groups: 1) BE, in which animals received binge-like intermittent access to PF daily for 2-hours; or 2) non-BE, in which animals received continuous (24-hour), non-intermittent access to PF. The BE group selected for this paradigm recapitulates key clinical diagnostic characteristics including intermittent consumption of a large amount of food which is consumed in a short period of time (American Psychiatric Association, 2013). While not a diagnostic requirement, food consumed during $B E$ is typically palatable in nature-i.e., high in sweetness and fat, but low in nutritional value (Drewnowski, Bellisle, Aimez, \& Remy, 1987; Gendall, Joyce, \& Abbott, 1999; Kales, 1990; Yanovski et al., 1992). In line with this, the PF used in the paradigm was sweetened condensed milk, diluted in a 3:1 ratio with water (Furlong et al., 2014). All mice had ad libitum access to standard chow throughout the paradigm and were never placed on food restriction.

Mice completed four weeks of daily PF feeding tests. Prior to each feeding test, mice were removed from group-housed conditions and placed in individual feeding test cages for the duration of each 2-hour feeding test. PF (delivered via $50 \mathrm{~mL}$ conical tube with sipper attachment) and standard chow were weighed at the beginning and end of the 2-hour feeding test, and body weight was measured daily. At the completion of each feeding test, mice were removed from their feeding test cages and returned to their group housed home cage. Animals in the non-BE group had a new bottle of PF placed in the home cage daily for continuous access to the PF. This bottle was weighed daily prior to the feeding test.

\section{Fiber Photometry Recordings and Processing}

All in vivo fiber photometry recordings took place in operant chambers (Med Associates) equipped with contact lickometers mounted to the chamber wall. Contact lickometers (Med 


\section{Corticostriatal dynamics underlying BE 6}

Associates) registering TTL outputs for each individual lick were used to identify feeding onset (first lick of a feeding bout) and feeding offset (last lick of a feeding bout). Bouts were defined as $\geq 2$ licks with breaks in between licking of no less than 3 seconds. Prior to the start of the study, all animals were habituated over multiple days to the operant chamber and the lickometer (containing only water). Animals were also habituated for at least two sessions to scruffing and cable attachment. There were two photometry recordings during the study, one at a baseline timepoint (prior to initiation of the BE paradigm) and a chronic timepoint (after four weeks of engagement in the BE paradigm). Animals were water restricted (and PF restricted for the nonBE group) overnight prior to recording days, while maintaining ad libitum access to standard chow. The first recording at the baseline timepoint occurred prior to initiation of the BE paradigm, and animals were therefore naïve to PF. This design capitalizes on the benefits of pre-clinical approaches by monitoring activity from first exposure/onset through chronic duration of behavior. The baseline recording was 40 minutes in duration to account for neophobia to the PF and assure there were enough feeding bouts for alignment of neural data. The second recording was 20 minutes in duration and took place at the chronic timepoint after completion of the BE paradigm (i.e., four weeks).

Photometry recordings were completed using a 3-color, 2-region system

(Neurophotometrics). Animals were connected to the system using a $4 \mathrm{x}$ branching cable (Doric) to allow for simultaneous recording from 2 regions in each animal. The system pulsed at $40 \mathrm{~Hz}$ and interleaved three LED channels $(415 \mathrm{~nm}, 470 \mathrm{~nm}, 560 \mathrm{~nm})$ during recordings. This resulted in detection of 1) an isosbestic signal, 2) GCaMP6m activity (DLS), and 3) jRCaMP1b activity (IL). Bonsai was used to interface the photometry system with the Med Associates TTL data and provide synchronization with the photometry signal in real time. After recordings, traces were separated to examine activity within each channel independently. All photometry data was processed and analyzed using custom MATLAB scripts. Processing began by removing motion artifacts from the 470 (GCaMP6m) and 560 (jRCaMP1b) channels using the 415 channel (isosbestic) trace as a reference point. Next, traces were corrected for exponential decay and run through a low-pass filter (order 6 , frequency 3 ). A moving minimum using a sliding minimum of 120 seconds was applied to remove large fluctuations from the traces. Finally, the traces were z-scored for normalization. These traces were aligned with the TTL outputs from the lickometers for analysis of neural activity at onset and offset of feeding bouts. For each trace, data were aligned to the behavior of interest at timepoint "0" (feeding onset, feeding offset), and trace analysis window included the three seconds prior to the event and one second after the event. 


\section{Histology}

After completion of the BE paradigm and photometry recordings, all mice were sacrificed via transcardial perfusion using 4\% paraformaldehyde (PFA). Brains were removed and remained in PFA for an additional 24 hours, cut into 35-micron sections, mounted onto slides, and cover slipped with DAPI mounting media. All sides were scanned using an Olympus slide scanning microscope. Location of fiber implant was identified by examining damage tracks in the tissue, and placement of the fiber and spread of the virus were used to include or exclude animals from analysis.

\section{Statistical Analyses}

For data from the BE paradigm, repeated measures ANOVAs (or mixed models in the case of missing data) with Bonferroni post-hoc analyses were used. Results are presented as mean \pm SEM. A p-value of $\leq 0.05$ was set to determine statistical significance. Statistical analyses were run using GraphPad Prism 9.

For fiber photometry data, multilevel spline regressions were run on the normalized photometry data during feeding bouts (group analysis of IL and DLS data) or the residuals of the best fitting model (exploratory variance analysis of DLS data). The best fitting models were chosen via model comparisons that employed the Bayesian information criterion (BIC), which considers the degree of flexibility (i.e., number of change points) of the model and penalizes more flexible models (Kuha, 2004). All models were a full factorial analysis, and all categorical predictors were effect coded. For all analyses, the residual distributions were examined for normality and homoscedasticity. All group analysis models met the assumptions, but the two variance analysis models were heteroscedastic. To satisfy the assumption of homoscedasticity, the residuals of the best fitting models were square root transformed prior to analysis. Predictors included the between-subjects categorical variable Group (BE, non-BE), and the within-subjects categorical variable Stage (Baseline, Chronic) and continuous variable Time (-3 seconds prior to event of interest to 1 second after the event of interest). The random effects structure included Intercept (subject) and two slopes (Stage and Time). Planned comparisons were run for significant interactions including Time, Stage, and Group using Type III Sums of Squares. All photometry data were analyzed using $R(R$ 4.0.3). The highest order significant interactions $(p<$ $0.05)$ including the variables Stage and Group were followed by planned comparisons using $R$ 's emmeans package. Group $\times$ Stage $\times$ Time interactions were followed by planned comparisons 
Corticostriatal dynamics underlying BE 8

at 500 ms intervals using emmeans. Results are presented as the model fit mean \pm SEM. Raw values alongside model fits are presented in the Extended Data Tables 1-4.

\section{Results}

\section{Binge Eating Paradigm}

All animals completed 4 weeks of the BE paradigm. On feeding test days, total intake of PF and chow during each daily feeding test was measured. Body weight was also measured daily. For PF intake, there was a significant Time $x$ Group interaction $(F(8.32,237.4)=31.60, p$

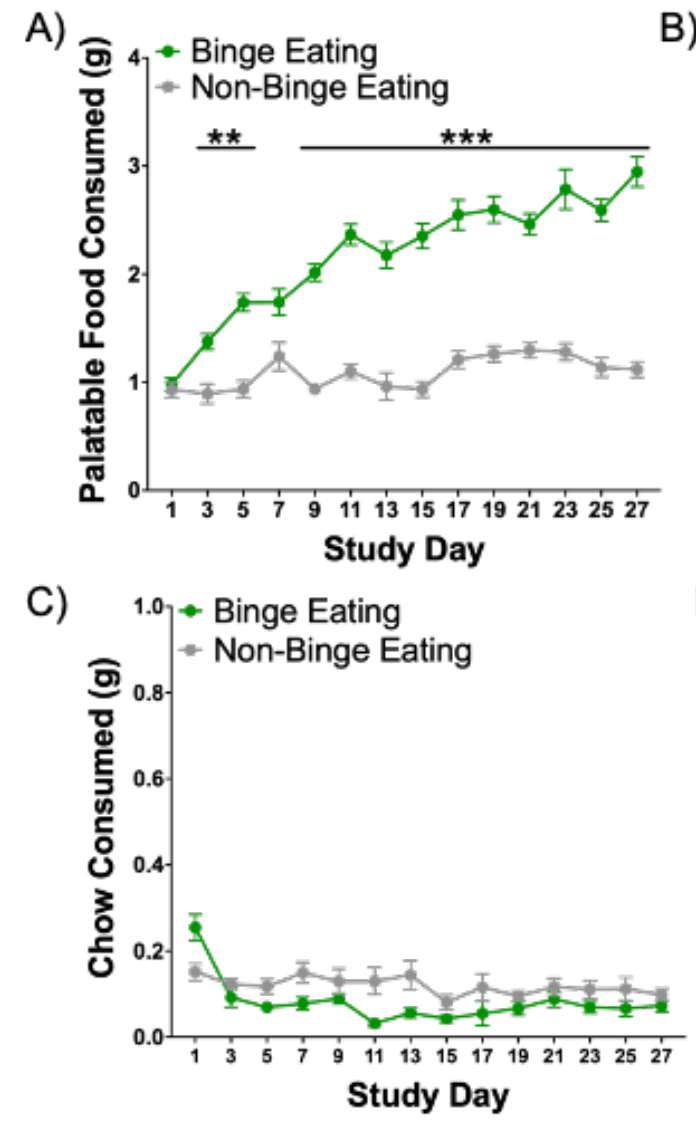

B)
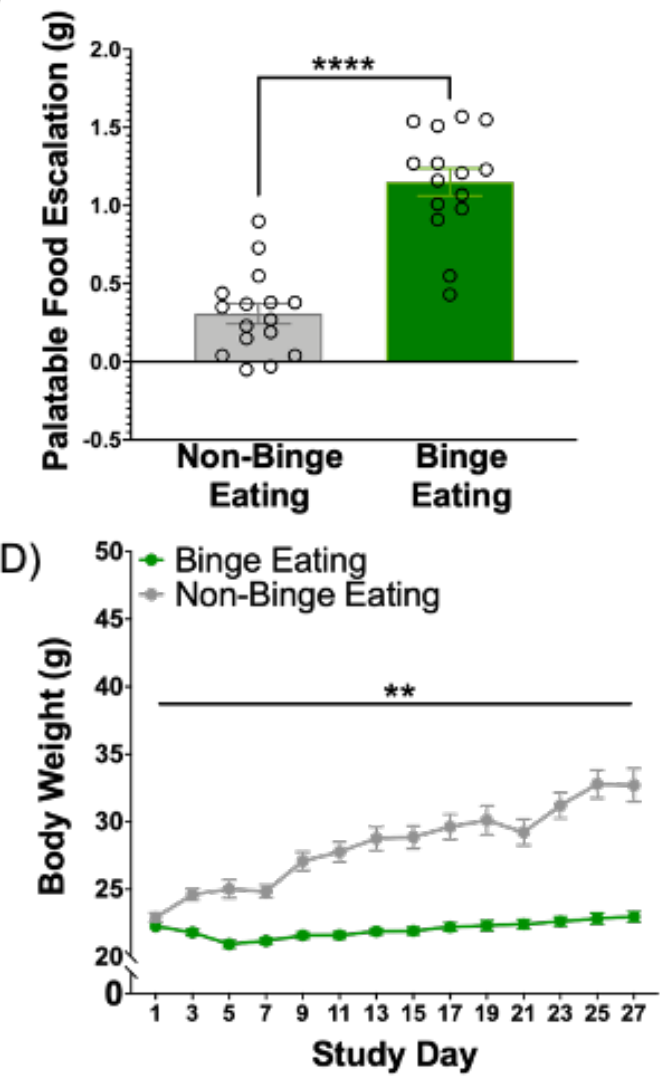

Figure 1. Binge eating paradigm behavioral data. $\mathrm{PF}$, chow, and BW data are graphed showing every other day. $\mathrm{N}=16 \mathrm{BE}, 16$ Non-BE. A) BE animals consumed significantly more PF compared to non-BE animals during feeding tests. B) Only BE animals escalated their PF intake across the study period. C) There were no specific days identified where chow intake was different between $\mathrm{BE}$ and non-BE mice. D) Body weight significantly increased in the non-BE group across the study period. ${ }^{\star \star} p<0.01,{ }^{\star \star \star} p<.001,{ }^{\star \star \star \star} p<.0001$.

$<.0001)$ such that BE animals consumed significantly more PF compared to non-BE mice during feeding tests starting on day two (Fig. 1A). This pattern persisted across the duration of the study (all $p s<.001$, except for Day 2, 3, $p<01$; Day 7, $p=.21$ ). Further evidence of differences in PF intake between groups was found across the four week BE paradigm such 
that BE animals escalated their PF intake during feeding tests significantly more than non-BE mice (Fig. $1 \mathrm{~B} ; \mathrm{t}=7.68 \mathrm{df}=29, p<.0001$ ). There was a significant Time $\times$ Group interaction for chow intake during feeding tests (Fig. 1C, F $(26,764)=2.67, p<.0001)$; however, post-hoc analysis identified no significant differences across days between $B E$ and non-BE mice. There was also a significant Time $x$ Group interaction for body weight (Fig. 1D, F (26, 780) = 50.65, $p$ $<.0001$ ) such that non-BE mice weighed significantly more compared to BE animals; this difference emerged on study day 2 and persisted across the study (all $p s<.01$ ).

\section{In Vivo Fiber Photometry - Infralimbic Cortex}

Onset. Comparing BICs, the best fitting model for IL feeding onset included five change points. There was no significant Group $\times$ Stage $\left(\chi^{2}(1)=0.05, p=0.83\right)$ or Group $\times$ Stage $\times$ Time $\left(\chi^{2}(5)=1.69, p=0.89\right)$ interaction. There were no differences in activity between or within $\mathrm{BE}$ and non-BE groups (Figs. 2A, 2B). Other lower effects are shown in Extended Data Table 1.
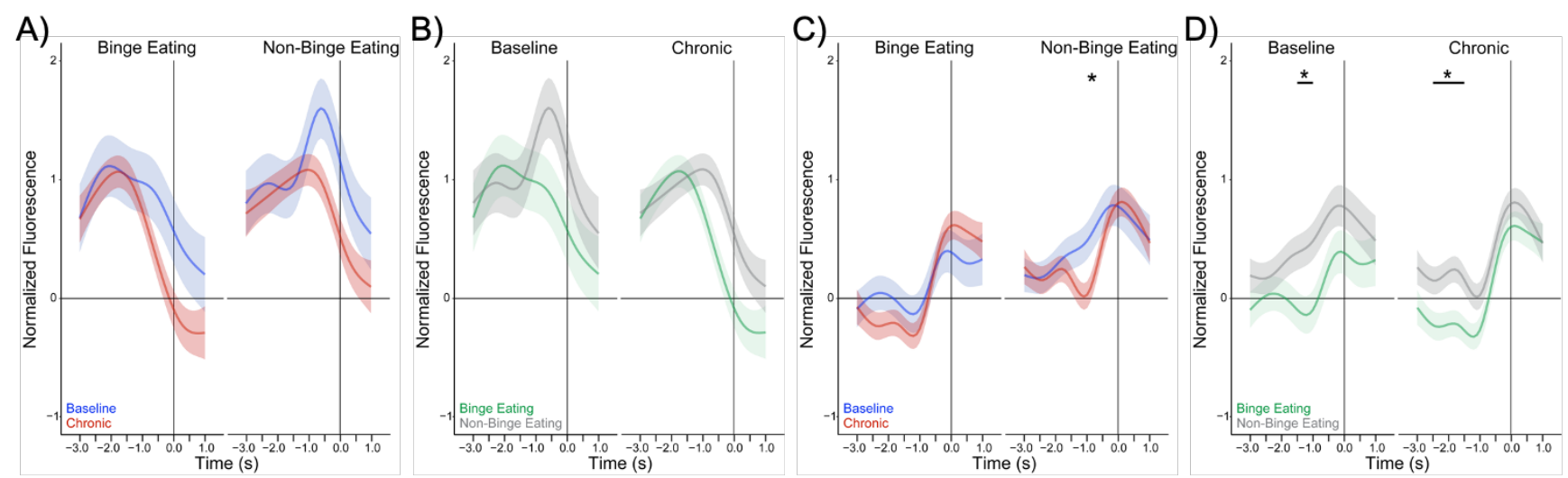

Figure 2. In vivo fiber photometry in the IL at feeding onset and offset. All data aligned to feeding onset $(A, B)$ or feeding offset $(C, D)$ at 0 . A) There were no differences within BE or non-BE groups in IL activity associated with feeding onset. $B$ ) There were no differences between $B E$ and non-BE mice in feeding onset IL activity. C) Non-BE animals had significantly lower activity at the chronic timepoint -1.0 seconds prior to feeding offset vs. baseline. D) At baseline, BE animals had significantly lower activity vs. non-BE animals -1.5 and 1.0 seconds before feeding offset. At the chronic timepoint, BE animals had significantly lower activity $-2.5,-2.0$, and -1.5 seconds before feeding offset vs. non-BE animals. ${ }^{*} p<$ 0.05 .

Offset. Comparing BICs, the best fitting model for IL feeding offset included six change points. There was a significant Group $\times$ Stage $\times$ Time interaction $\left(\chi^{2}(5)=26.24, p<0.01\right)$. The interaction revealed that the non-BE group had reduced activity compared to baseline at -1.0 ( $z$ $=2.42, p=0.02$ ) prior to feeding offset (Fig. $2 \mathrm{C}$ ). There were no differences within the BE group (all $p>0.10)$. At baseline, BE animals had lower activity than the non-BE group at -1.5 ( $z$ $=-2.27, p=0.03)$ and $-1.0(z=-2.57, p=0.02)$ seconds prior to feeding offset (Fig. 2D). 
Compared to the non-BE group, BE animals had lower activity at $-2.5(z=-2.27, p=0.03),-2.0$ $(z=-2.85, p<0.01)$, and $-1.5(z=-2.75, p=0.01)$ seconds at the chronic timepoint (Fig. 2D). However, these results at the chronic duration are inconclusive given the significant differences in baseline activity between groups (Fig. 2D, left panel). Other lower effects are shown in Extended Data Table 2.

\section{In Vivo Fiber Photometry - Dorsolateral Striatum}

Feeding Onset. Comparing BICs, the best fitting model for DLS feeding onset included five change points. There was a significant Group $\times$ Stage $\times$ Time interaction $\left(\chi^{2}(5)=41.17, p<\right.$ $0.01)$. The BE group had reduced DLS activity at the chronic timepoint compared to baseline at $-2.5(z=2.13, p=0.03)$ and $-2.0(z=2.16, p=0.03)$ seconds prior to feeding onset (Fig. $3 \mathrm{~A})$. There were no significant differences between the baseline and chronic timepoints in non-BE animals (Fig. 3A). Between group analysis showed that, compared to the non-BE group, BE animals had reduced activity from -2.5 to -1.0 seconds $\left(z_{\mathrm{s}}=-4.46\right.$ to -2.72 , all $\left.p<0.01\right)$ prior to feeding bout onset at the chronic timepoint (Fig. 3B). Other lower effects can be found in Extended Data Table 1. Exploratory analyses examining variance across groups found a significant Group $\times$ Stage interaction $\left(\chi^{2}(1)=4.32, p=0.04\right)$. The BE group had reduced variability in their activity related to feeding onset at the chronic timepoint compared to baseline $(z=2.29, p=0.02)$ (Fig. $3 \mathrm{C})$, while there were no changes in variance in the non-BE group $(z=$ $-1.27, p=0.21$ ) (Fig. 3C). The interaction also showed that at the chronic timepoint, the $B E$ group had lower variability than the non-BE group $(z=-2.34, p=0.02)$, but not during the baseline timepoint ( $z=1.17, p=0.24)$ (Fig. 3C).

Feeding Offset. Comparing BICs, the best fitting model for DLS feeding offset included five change points. There was a significant Group $\times$ Stage $\times$ Time interaction $\left(\chi^{2}(5)=30.51, p<\right.$ 0.01). The BE group had reduced activity in the DLS at the chronic timepoint compared to baseline at $-1.5(z=1.97, p=0.048)$ and $-1.0(z=2.33, p=0.02)$ seconds prior to feeding offset (Fig. 3D). The non-BE group had reduced activity at the chronic timepoint compared to baseline at $-2.5(z=-2.13, p=0.03)$ seconds (Fig. 3D). Compared to the non-BE group, BE animals trended towards having reduced activity at $-0.5(z=-1.80, p=0.07)$ and $0(z=-1.76, p=0.08)$ seconds prior to feeding bout offset at the chronic, but not baseline, timepoint (Fig. 3E). Other lower effects are shown in Extended Data Table 2. Exploratory analyses examining variance in DLS activity associated with feeding offset found a significant Group $\times$ Stage interaction $\left(\chi^{2}(1)=\right.$ $5.64, p=0.02)$. The interaction revealed no within group differences between timepoints, but 
bioRxiv preprint doi: https://doi.org/10.1101/2021.11.04.467011; this version posted November 5, 2021. The copyright holder for this preprint (which was not certified by peer review) is the author/funder, who has granted bioRxiv a license to display the preprint in perpetuity. It is made available under aCC-BY-NC-ND 4.0 International license.

Corticostriatal dynamics underlying BE 11

showed that at the chronic timepoint, the BE group had lower variability than the non-BE group $(z=-2.39, p=0.02)$, but not during the baseline timepoint $(z=0.08, p=0.94)$ (Fig. 3F).
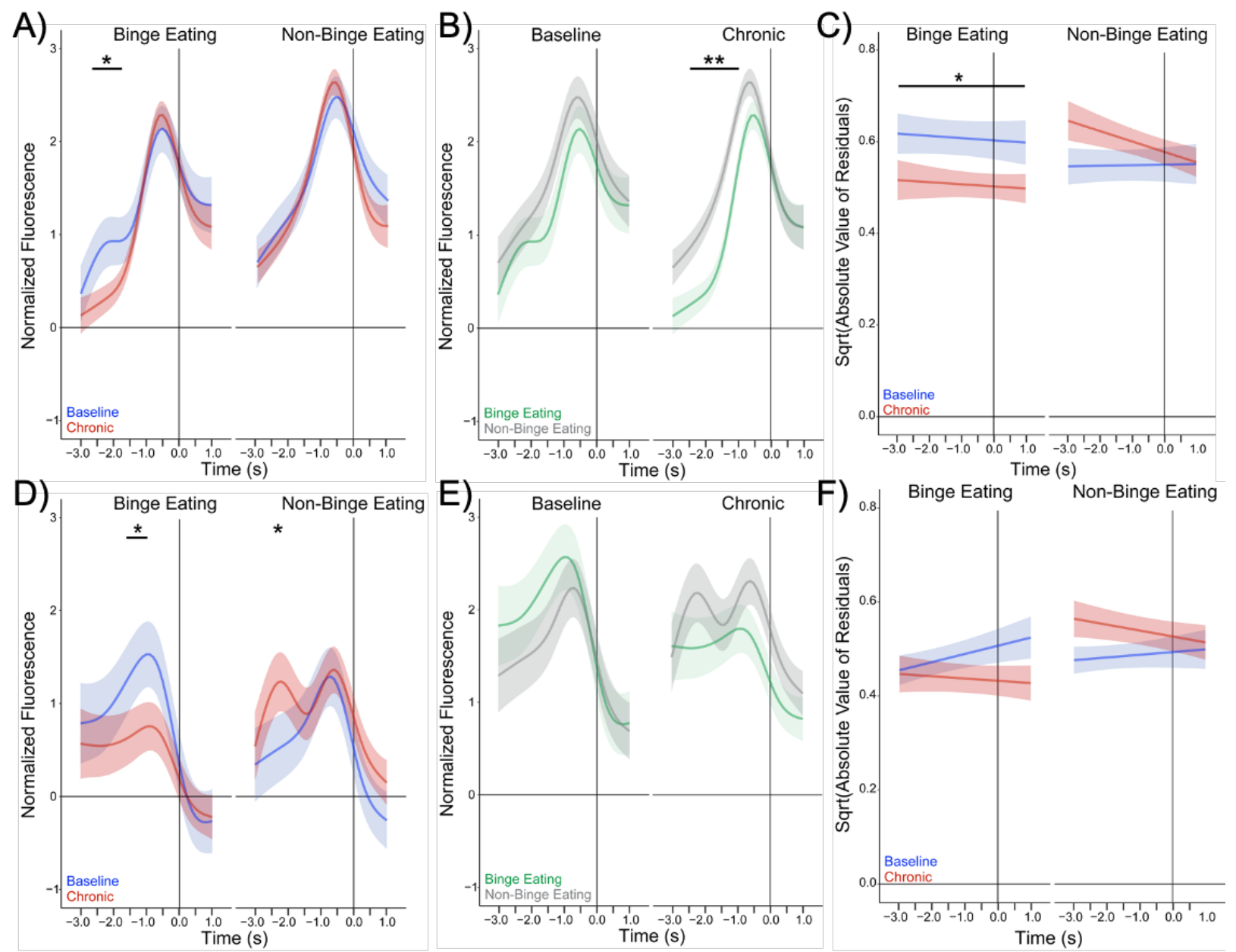

Figure 3. In vivo fiber photometry in the DLS at feeding onset and offset. All data aligned to feeding onset $(A, B, C)$ or feeding offset $(D, E, F)$ at 0 . A) BE had significantly lower activity at chronic vs. baseline at -2.5 and -2.0 seconds prior to feeding onset. $B$ ) $B E$ animals had significantly lower activity vs. non-BE animals at the chronic timepoint -2.5 to -1.0 seconds prior to feeding onset. $C$ ) Variance in activity associated with feeding onset significantly decreased from the acute to chronic in $\mathrm{BE}$, but not non-BE mice. Variance at chronic timepoint was lower in BE vs. non-BE mice. Group $\times$ Stage interaction $(p=0.04)$. D) BE mice had significantly lower activity at chronic vs. baseline at -1.5 and -1.0 seconds prior to feeding offset. Non-BE mice had significantly lower activity at chronic vs. baseline at -2.5 seconds. E) Trend toward significant difference at chronic timepoint with lower BE activity at -0.5 and 0.0 seconds before feeding offset vs. non-BE mice. F) No differences in variance related to feeding offset in $B E$ and non-BE mice. ${ }^{*} p<0.05$, ${ }^{* *} p<0.01$.

\section{Discussion}

This study used in vivo fiber photometry to investigate longitudinal changes in neural activity within corticostriatal circuitry in BE and non-BE mice. We examined activity during naturalistic binge-like eating episodes aligned to specific feeding behaviors (feeding onset, 
feeding offset). While findings in the IL showed no specific changes related to BE (Fig. 2), we found differential activity patterns in the DLS after a chronic duration of PF intake (Fig. 3). Together, these findings suggest reduced recruitment of DLS is specific to animals with a history of binge-like eating (i.e., intermittent) and not general (i.e., continuous) PF intake. These results point to a specific neural mechanism in the DLS underlying chronic BE, highlighting a potential target for future treatment intervention.

Previous work has suggested that BE leads to an imbalance in goal-directed and habitual behavior. Baseline deficits in goal-directed responding in rats were associated with higher levels of subsequent binge-eating upon exposure to PF (LeMon, Sisk, Klump, \& Johnson, 2019), suggesting that impaired goal-directed responding may be a risk factor for BE development. Other work has shown that rats with a history of BE engaged in more lever pressing during outcome devaluation testing, suggesting BE led to more habitual responding compared to continuous access or control rats (Furlong et al., 2014). This pattern of habitual responding was associated with increased outcome-devaluation-test-related-c-Fos activity in areas associated with habit (i.e., DLS, IL, Furlong et al., 2014). Given our findings of decreased DLS activity after chronic BE at feeding onset and offset, this may suggest that BE episode feeding behaviors are not driven by habit. Clinical research of BE populations has found that when viewing images of PF, individuals with $B E$ show increased recruitment of goal-directed brain regions (e.g., ventromedial prefrontal cortex, Neveu et al., 2018). However, on a decision making task unrelated to food, individuals with $B E$ disorder engaged in significantly more habitual responding (Voon et al., 2015). Together, findings suggest that feeding and food related tasks may differently recruit goal-directed/habitual circuitry compared to tasks assessing habitual decision-making (unrelated to feeding and food) in individuals with BE. Future work should investigate engagement of corticostriatal regions associated with goal-directed behavior (e.g., prelimbic cortex, dorsomedial striatum) during binge-like eating, as this would help disentangle the relationship between goal-directed and habitual behavior in the control of pathologically feeding behavior in BE.

Feeding bouts involve a repetitive sequence of behaviors including feeding onset, consumption, and feeding offset, and previous work has shown that the DLS plays a key role in initiating and completing sequences. Training on a 2-step lever pressing task showed that lesions of the DLS impaired acquisition of correct sequence completion in mice (Yin, 2010), and work using a viral strategy to specifically suppress activity of D1+ spiny projection neurons in the DLS found impairments in sequence accuracy (Rothwell et al., 2015). We measured DLS activity during distinct phases of the feeding sequence (feeding onset, feeding offset). At both 
feeding onset and offset, BE animals showed a reduction in DLS activity from the baseline to the chronic timepoint. At the chronic timepoint, BE animals also had lower activity compared to non-BE animals at feeding onset (Fig. 3B). At feeding offset, BE mice showed a blunting of activity from baseline to the chronic timepoint (Fig. 3D) and trended toward being significantly lower than non-BE mice at the chronic timepoint (Fig. 3E). Our findings showing decreased/blunted DLS activity in BE mice may be consistent with difficulty in executing feeding sequences in BE mice.

Reduced activity in the DLS of BE mice may result from decreased activity in upstream regions that project to the DLS and contribute to sequence performance, such as the secondary motor cortex (M2), a primary input to the DLS (Lee et al., 2019; Rothwell et al., 2015). Pharmacological inactivation of $\mathrm{M} 2$ in rats with a history of BE increased PF consumption (Corwin et al., 2016), suggesting that lower M2 activity may contribute to impairments in feeding offset/feeding sequence completion. M2 $\rightarrow$ DLS projections are critical for correct execution and termination of behavioral sequences (Rothwell et al., 2015). Therefore, the blunted activity found at feeding onset in the DLS of BE mice may represent less activity from M2 $\rightarrow$ DLS projections in initiating feeding, promoting ongoing repetitive action. While these data underscore the importance of the DLS and associated inputs in generating sequenced behavior, future work should use alternative sequence testing paradigms in chronic BE and non-BE mice to compare within-BE to within-task DLS activity. Additionally, manipulation of M2 $\rightarrow D L S$ projections during distinct phases of the feeding sequence would directly dissect the role of these projections in binge-like eating.

In our current work, we were able to capitalize on pre-clinical approaches to longitudinally examine in vivo neural activity underlying feeding behaviors during binge-like eating. Our results point to a specific neural mechanism in the DLS underlying chronic BE, which highlights a potential target for future development of targeted biologically informed treatment interventions. Future work should further investigate corticostriatal contributions to $B E$ behavior and the interplay between goal-directed and habitual behavior. 


\section{References}

American Psychiatric Association. (2013). Diagnostic and Statistical Manual of Mental Disorders (5th ed.). Arlington, VA: American Psychiatric Association.

Burguiere, E., Monteiro, P., Mallet, L., Feng, G., \& Graybiel, A. M. (2015). Striatal circuits, habits, and implications for obsessive-compulsive disorder. Current Opinion in Neurobiology, 30, 59-65.

Conti, C., Lanzara, R., Scipioni, M., lasenza, M., Guagnano, M. T., \& Fulcheri, M. (2017). The relationship between binge eating disorder and suicidality: A systematic review. Frontiers in Psychology, 8, 2125. doi: 10.3389/fpsyg.2017.02125

Corwin, R. L., Wojnicki, F. H. E., Zimmer, D. J., Babbs, R. K., McGrath, L. E., Olivos, D. R., . . Hayes, M. R. (2016). Binge-type eating disrupts dopaminergic and GABAergic signaling in the prefrontal cortex and ventral tegmental area. Obesity, 24, 2118-2125.

Culbert, K. M., Sinclair, E. B., Hildebrandt, B. A., Sisk, C. L., \& Klump, K. L. (2018). Perinatal testosterone contributes to mid-to-post pubertal sex differentiated risk for binge eating in male and female rats. Journal of Abnormal Psychology, 127, 239-250. doi: 10.1037/abn0000334

Drewnowski, A., Bellisle, F., Aimez, P., \& Remy, B. (1987). Taste and bulimia. Physiology \& Behavior, 41, 621-626. doi: 10.1016/0031-9384(87)90320-9

Furlong, T. M., Jayaweera, H. K., Balleine, B. W., \& Corbit, L. H. (2014). Binge-like consumption of a palatable food accelerates habitual control of behavior and is dependent on activation of the dorsolateral striatum. Journal of Neuroscience, 34, 5012-5022. doi: 10.1523/JNEUROSCI.3707-13.2014

Gendall, K. A., Joyce, P. R., \& Abbott, R. M. (1999). The effects of meal composition on subsequent craving and binge eating. Addictive Behaviors, 24, 305-315. doi: 10.1016/S0306-4603(98)00046-X 
George, O., \& Koob, G. F. (2010). Individual differences in prefrontal cortex function and the transition from drug use to drug dependence. Neuroscience \& Biobehavioral Reviews, 35, 232-247.

Gillan, C. M., Papmeyer, M., Morein-Zamir, S., Sahakian, B. J., Fineberg, N. A., Robbins, T. W., \& de Wit, S. (2011). Disruption in the balance between goal-directed behavior and habit learning in obsessive-compulsive disorder. American Journal of Psychiatry, 168, 718726.

Goracci, A., di Volo, S., Casamassima, F., Bolognesi, S., Benbow, J., \& Fagiolini, A. (2015). Pharmacotherapy of binge-eating disorder: a review. Journal of Addiction Medicine, 9, 119.

Graybiel, A. M., \& Rauch, S. L. (2000). Toward a neurobiology of obsessive-compulsive disorder. Neuron, 28, 343-347.

Harrison, B. J., Soriano-Mas, C., Pujol, J., Ortiz, H., López-Solà, M., Hernández-Ribas, R., . . . Pantelis, C. (2009). Altered corticostriatal functional connectivity in obsessivecompulsive disorder. Archives of General Psychiatry, 66, 1189-1200.

Herpertz, S., Albus, C., Wagener, R., Kocnar, M., Wagner, R., Henning, A., . . Thomas, W. (1998). Comorbidity of Diabetes and Eating Disorders: Does diabetes control reflect disturbed eating behavior? Diabetes Care, 21, 1110-1116. doi:

10.2337/diacare.21.7.1110

Hogarth, L., Balleine, B. W., Corbit, L. H., \& Killcross, S. (2013). Associative learning mechanisms underpinning the transition from recreational drug use to addiction. Annals of the New York Academy of Sciences, 1282, 12-24.

Horstmann, A., Dietrich, A., Mathar, D., Pössel, M., Villringer, A., \& Neumann, J. (2015). Slave to habit? Obesity is associated with decreased behavioural sensitivity to reward devaluation. Appetite, 87, 175-183. 
Janssen, L. K., Duif, I., van Loon, I., Wegman, J., de Vries, J. H. M., Cools, R., \& Aarts, E. (2017). Loss of lateral prefrontal cortex control in food-directed attention and goaldirected food choice in obesity. Neurolmage, 146, 148-156.

Kales, E. F. (1990). Macronutrient analysis of binge eating in bulimia. Physiology \& Behavior, 48, 837-840. doi: 10.1016/0031-9384(90)90236-W

Kalivas, P. W. (2008). Addiction as a pathology in prefrontal cortical regulation of corticostriatal habit circuitry. Neurotoxicity Research, 14, 185-189.

Keel, P. K., Dorer, D. J., Franko, D. L., Jackson, S. C., \& Herzog, D. B. (2005). Postremission predictors of relapse in women with eating disorders. American Journal of Psychiatry, 162, 2263-2268. doi: 10.1176/appi.ajp.162.12.2263

Kessler, R. M., Hutson, P. H., Herman, B. K., \& Potenza, M. N. (2016). The neurobiological basis of binge-eating disorder. Neuroscience \& Biobehavioral Reviews, 63, 223-238. doi: 10.1016/j.neubiorev.2016.01.013

Klump, K. L., Culbert, K. M., \& Sisk, C. L. (2017). Sex differences in binge eating: Gonadal hormone effects across development. Annual Review of Clinical Psychology, 13, 183207.

Klump, K. L., Racine, S. E., Hildebrandt, B., \& Sisk, C. L. (2013). Sex differences in binge eating patterns in male and female adult rats. International Journal of Eating Disorders, 46, 729-736. doi: 10.1002/eat.22139

Kuha, J. (2004). AIC and BIC: Comparisons of assumptions and performance. Sociological methods \& research, 33, 188-229.

Lee, K., Bakhurin, K. I., Claar, L. D., Holley, S. M., Chong, N. C., Cepeda, C., .. Masmanidis, S. C. (2019). Gain modulation by corticostriatal and thalamostriatal input signals during reward-conditioned behavior. Cell Reports, 29, 2438-2449. e2434. 
LeMon, J. V., Sisk, C. L., Klump, K. L., \& Johnson, A. W. (2019). Reduced sensitivity to devaluation for instrumental but not consummatory behaviors in binge eating prone rats. Physiology \& Behavior, 206, 13-21.

Moore, C. F., Sabino, V., Koob, G. F., \& Cottone, P. (2016). Pathological Overeating: Emerging Evidence for a Compulsivity Construct. Neuropsychopharmacology, 1-15. doi: 10.1038/npp.2016.269

Neveu, R., Neveu, D., Carrier, E., Gay, A., Nicolas, A., \& Coricelli, G. (2018). Goal directed and self-control systems in bulimia nervosa: an fMRI study. EBioMedicine, 34, 214-222.

Olmsted, M. P., MacDonald, D. E., McFarlane, T., Trottier, K., \& Colton, P. (2015). Predictors of rapid relapse in bulimia nervosa. International Journal of Eating Disorders, 48, 337-340. doi: 10.1002/eat.22380

Pearson, C. M., Wonderlich, S. A., \& Smith, G. T. (2015). A risk and maintenance model for bulimia nervosa: From impulsive action to compulsive behavior. Psychological review, $122,516$.

Rothwell, P. E., Hayton, S. J., Sun, G. L., Fuccillo, M. V., Lim, B. K., \& Malenka, R. C. (2015). Input-and output-specific regulation of serial order performance by corticostriatal circuits. Neuron, 88, 345-356.

Spitzer, R. L., Yanovski, S., Wadden, T., Wing, R., Marcus, M. D., Stunkard, A., . . Horne, R. L. (1993). Binge eating disorder: its further validation in a multisite study. International Journal of Eating Disorders, 13, 137-153. doi: 10.1002/1098108X(199303)13:2<137::AID-EAT2260130202>3.0.CO;2-\%23

Stice, E., Cameron, R. P., Killen, J. D., Hayward, C., \& Taylor, C. B. (1999). Naturalistic weightreduction efforts prospectively predict growth in relative weight and onset of obesity among female adolescents. Journal of Consulting and Clinical Psychology, 67, 967-974. doi: 10.1037//0022-006x.67.6.967 
Stice, E., Presnell, K., \& Spangler, D. (2002). Risk factors for binge eating onset in adolescent girls: a 2-year prospective investigation. Health Psychology, 21, 131-138. doi:

10.1037/0278-6133.21.2.131

Telch, C. F., \& Stice, E. (1998). Psychiatric comorbidity in women with binge eating disorder: Prevalence rates from a non-treatment-seeking sample. Journal of Consulting and Clinical Psychology, 66, 768-776. doi: 10.1037//0022-006x.66.5.768

Vocks, S., Tuschen-Caffier, B., Pietrowsky, R., Rustenbach, S. J., Kersting, A., \& Herpertz, S. (2010). Meta-analysis of the effectiveness of psychological and pharmacological treatments for binge eating disorder. International Journal of Eating Disorders, 43, 205217.

Voon, V., Baek, K., Enander, J., Worbe, Y., Morris, L. S., Harrison, N. A., . . Daw, N. (2015). Motivation and value influences in the relative balance of goal-directed and habitual behaviours in obsessive-compulsive disorder. Translational Psychiatry, 5, e670.

Yanovski, S. Z., Leet, M., Yanovski, J. A., Flood, M., Gold, P. W., Kissileff, H. R., \& Walsh, B. T. (1992). Food selection and intake of obese women with binge-eating disorder. The American Journal of Clinical Nutrition, 56, 975-980. doi: 10.1093/ajcn/56.6.975 Yin, H. H. (2010). The sensorimotor striatum is necessary for serial order learning. Journal of Neuroscience, 30, 14719-14723. 